\title{
DIE DEUTSCHE ALLGEMEINE ZEITUNG: ANMERKUNGEN ZUR SPRACHE DER KASACHSTANDEUTSCHEN PRESSE
}

\section{ANLIEGEN UND ZIELE}

Die auslandsdeutsche Presse ist bisher kaum ins Blickfeld sprachwissenschaftlicher Forschungsarbeiten gerückt, obwohl sie vor allem wegen ihrer spezifischen (inter-)kulturellen und (mehrsprachigen) kommunikativen Einbettung viel Potenzial bietet. Die Deutsche Allgemeine Zeitung (im Weiteren: DAZ) zeichnet sich durch besonders aufschlussreiche Rahmenbedingungen aus. Denn ihr Alleinstellungsmerkmal besteht in der hohen Komplexität ihrer sprachlich-kulturellen Hintergrundkonstellationen in einem kulturell mehrfach kodierten Raum: Sie ist ein deutschsprachiges Medium in einem kasachischen Umfeld, wobei die meisten ihrer einheimischen Leser(innen), einschließlich der Kasachstandeutschen, Russisch als dominierende Erstsprache verwenden.

In diesem Kontext setzt sich der vorliegende Aufsatz zwei Ziele. ${ }^{1}$ In erster Linie soll er im Horizont der interkulturellen Linguistik unter Rückgriff auf qualitative hermeneutisch-analytische Verfahren signifikante Profilmerkmale der DAZ als auslandsdeutsche Zeitung erschließen. Außerdem wird auch ein Beitrag zur Herausarbeitung von Phänomenen der Kulturalität im behandelten Pressediskurs durch Exponierung kulturinduzierter - vor allem lexikalischer - Merkmale angestrebt. Dies soll nicht mit einem normativen fehleranalytischen Ansatz, sondern vielmehr in einem deskriptiven, vor allem kontaktund interkulturalitätsorientierten, linguistischen Denkrahmen erfolgen. ${ }^{2}$ Dabei geht es aus Gründen des Umfangs um die Veranschaulichung der Typenvielfalt und Variabilität der eruierten Phänomene, nicht um eine Auflistung möglichst zahlreicher Belege.

\section{DIE DAZ ALS REFLEXIONSOBJEKT UND IHRE LEBENSWELTLICHE VERORTUNG}

Die DAZ ist eine Wochenzeitung in Kasachstan, die in deutscher und in russischer Sprache mit einer Auflage von bis zu 2.000 Exemplaren $^{3}$ (Quelle: DAZ 2018) im A3-For-

* foeldes@foeldes.eu; www.foeldes.eu

1 Der Beauftragten der Bundesregierung für Kultur und Medien (BKM) gilt für die Unterstützung des Projekts ein herzliches Dankeschön.

2 Jedoch können die Ausführungen in gewisser Hinsicht auf bestimmte normative Aspekte nicht verzichten, es werden ja Abweichungen von der „standarddeutschen Norm“ erfasst und dann kontaktlinguistisch interpretiert. Hierzu ist allerdings anzumerken, dass es im Deutschen als plurizentrische Sprache keine einheitliche Standardsprache und damit auch keine einheitliche Norm gibt.

3 Im Fließtext desselben Berichts wird die Auflage mit 1.000 beziffert (DAZ 2018). 
mat erscheint. Sie richtet sich vornehmlich an Angehörige der deutschen Minderheit in Mittelasien, aber auch an Sprachlernende und an deutschsprachige Tourist(inn)en und Firmenentsandte. Weiterhin wird auch das russischsprachige Publikum - des Deutschen nicht mehr lesekundige Kasachstandeutsche ${ }^{4}$ und Interessierte aus anderen ethnischen Gruppen - angesprochen. So behandelt das Medium auf zwölf Seiten Themen wie Politik, Wirtschaft und Kultur und informiert über Ereignisse im Zusammenhang mit der kasachstandeutschen Minderheit. Zu ihren Schwerpunktthemen gehören also die deutsch-kasachstanischen ${ }^{5}$ Beziehungen, Tendenzen in Politik, Kultur und den Gesellschaften Zentralasiens und Deutschlands sowie Perspektiven und Ausbildungsmöglichkeiten für die Jugend (Quelle: DAZ 2018). Als bilinguale Zeitung vom Typ komplementäre Mehrsprachigkeit ${ }^{6}$ konstituiert sie sich aus deutsch- und russischsprachigen Artikeln, wobei die erste Seite dominant deutsche Texte enthält. Zur Sicherstellung des sprachlichen Niveaus übernimmt außerdem das Auswärtige Amt über das Institut für Auslandsbeziehungen (ifa) die Kosten für eine Redaktionsstelle eines bundesdeutschen Journalisten. Zusätzlich zu dieser Stelle, die aktuell mit einer ifa-Redakteurin besetzt ist, besteht die Redaktion aus einer kasachstandeutschen Chefredakteurin und einer technischen Redakteurin. Da die Redaktionsabteilungen unabhängig voneinander an den Artikeln arbeiten, gibt es für den deutsch- und den russischsprachigen Teil jeweils einen zuständigen Leiter. Die Leitung des deutschsprachigen Teils übernimmt die ifa-Redakteurin. Sie trägt dabei die Verantwortung für die sprachliche Qualität der Artikel und entscheidet, welche Themen behandelt bzw. welche Texte abgedruckt werden. Die meisten Autor(inn)en der DAZ sind aus Zentralasien und verfügen über ein Sprachniveau von mindestens B1 oder sind deutsche Muttersprachler. Weiterhin wirken regelmäßig Praktikant(inn)en aus dem deutschen Sprachraum mit. ${ }^{7}$

Zur Historie der DAZ ist erwähnenswert, dass sie als Zeitung des Zentralkomitees der Kommunistischen Partei Kasachstans ab dem 1. Januar 1966 täglich (außer sonntags) unter dem Namen „Freundschaft“ herauskam. Mit dem Ende der kommunistischen Epoche stellte sich der Erscheinungsrhythmus in den 1990er Jahren um und die Zeitung erschien nur noch wöchentlich. Anfang des 21. Jahrhunderts übernahm die „Assoziation der gesellschaftlichen Vereinigung der Deutschen Kasachstans“ die Herausgabe der Zeitung, um deren Erhalt und Zukunft zu sichern (AGDM 2018).

4 Die Minderheitengruppe selbst betrachtet sich traditionell als ,russlanddeutsch“.

5 Mit dieser Formulierung gibt der Beitrag die in Kasachstan übliche Sprachverwendung wieder: Das Adjektiv kasachstanisch inkludiert alles, was mit Kasachstan zu tun hat, während kasachisch im engeren, ethnischen, Sinne verstanden wird.

6 Urbán (2017: 245ff.) unterscheidet diesbezüglich „simultane Mehrsprachigkeit“ (d. h. die Wiedergabe gleicher Inhalte in verschiedenen Sprachen, grundsätzlich für eine Zielgruppe von Lesern, die nur eine der Publikationssprachen beherrschen) einerseits und „komplementäre Mehrsprachigkeit"“ (d. h. verschiedene Inhalte in verschiedenen Sprachen, grundsätzlich für eine Zielgruppe multilingualer Leser) andererseits.

7 Der ifa-Redakteurin Othmara Glas, die diese Informationen am 16.06.2018 per E-Mail zur Verfügung stellte, sowie meiner BA-Studentin, Anne-Kathrin Sorg, die mich bei den Recherchen unterstützte, sei herzlich gedankt. 


\section{MATERIALBASIS}

Die durchgeführte datenorientierte empirische Analyse konzentriert sich auf die zum Zeitpunkt der Materialerfassung in Deutschland zugänglichen Printausgaben des Jahrgangs 2017. Fokussiert wird auf die deutschsprachigen Seiten dieses Jahrgangs; der russischsprachige Teil gehört nicht zum Gegenstandsfeld. Die ifa-Bibliothek in Stuttgart verfügt derzeit über die Ausgaben von Januar bis Mai. Im Bestand der Martin-Opitz-Bibliothek in Herne als der zentralen Bibliothek zur deutschen Kultur und Geschichte im östlichen Europa (einschließlich auch der asiatischen Teile der ehemaligen Sowjetunion) befinden sich lediglich einzelne Exemplare der Jahre 1995-2000, 2002 und 2006.

\section{BESCHREIBUNGSSCHWERPUNKT SALIENZEN}

\section{1}

Die vorliegende Studie richtet sich am Salienz-Konzept ${ }^{8}$ - „eine Aufmerksamkeit erzeugende Eigenschaft von Objekten“(Parkinson 2014: 92) - aus, das in der germanistischen Linguistik vorrangig in der Dialektologie verankert ist. ${ }^{9}$,Salienz' stellt also, wie auch im Aufsatz von Földes (2008a: 45ff.), ein analytisches Beschreibungsinstrument zur Identifizierung von Auffälligkeiten dar, die sich auf der Folie „binnendeutscher"10 Zeitungssprache registrieren lassen. ${ }^{11} \mathrm{Da}$ Salienz „von wahrnehmungsbezogenen Aspekten [...] wie der Sensibilität des Betrachters" abhängt (Parkinson 2014: 92), kommt eine Festlegung, was mit dem Label „Auffälligkeit“ zu markieren ist und was nicht, ohne eine gewisse Subjektivität nicht aus. Zur Erhöhung der Validität wurden daher in Ergänzung und als Korrektiv zu sprachlichen und kulturellen Kompetenzen des Verfassers im Hinblick auf Deutsch und Russisch sowie auf Kasachstanbezogenes mehrere bundesdeutsche und russisch- sowie kasachischsprachige Gewährspersonen, letztere sowohl aus Kasachstan als auch aus Russland, herangezogen. ${ }^{12}$ Hinsichtlich der eingesetzten methodologischen Strategien bzw. Verfahren der induktiven Untersuchung

8 Mit dem aus der Psychologie stammenden Konstrukt „Salienz“ arbeiten im Bereich der Sprachwissenschaft z. B. Lenz (2010) vornehmlich unter dem Gesichtspunkt der Dialektologie und Rácz (2013) unter dem der Soziolinguistik. Vorliegender Beitrag orientiert sich grundsätzlich an der Salienz-Konzeption von Purschke (2011: 84).

9 Letzten Endes könnte man etwas vereinfachend von (kognitiven) „Auffälligkeiten“ sprechen. Ein Gros der Manifestationen von Salienz ließe sich aus Sicht der Fremdheitsforschung als „Xenismus“ betrachten (vgl. Földes 2018b: 123ff.).

10 Mit „Binnendeutsch“ wird die standardorientierte Sprachverwendung im zusammenhängenden deutschen Sprach- und Kulturraum (Deutschland, Österreich und die Schweiz) gemeint, als Gegenpart zu sprachkommunikativen Praktiken deutschsprachiger Textproduzenten im nichtdeutschsprachigen Ausland.

11 Das heißt, der Deutschland-deutsche Standard galt operational als Bezugsgröße (nicht aber als Wertprämisse).

12 Allen voran sei Svetlana Frolova, Tanja Seliazneva, Anargul Zhumakhanova, Markus Hartmann und Johannes Steudel herzlich gedankt. 
sind vor allem (1) die fragestellungsbezogene Kollektion ${ }^{13}$ (Sammlung von Vorkommen eines bestimmten Phänomens), (2) die Typenbildung und (3) die Sequenzanalyse zu erwähnen. Die Studie fokussiert auf eine diskursorientierte Mikroebene und geht auf die Makroebene (z. B. Akteure und Netzwerke) nicht ein.

Anhand der empirischen Sichtung der Datengrundlage tat sich eine Bandbreite von Salienzen zum einen primär sprachbezogener und zum anderen primär kulturbezogener Natur auf, zwischen denen es mitunter fließende Übergänge gibt.

\section{2}

Dabei machen die Salienzen primär sprachbezogener Provenienz auf verschiedenen Ebenen die in quantitativer Hinsicht zentrale paradigmatische Phänomenklasse aus.

\subsection{1}

Die Studie konnte ein breites Spektrum (mono-)lexematischer Auffälligkeiten - einschließlich Aspekte der Semantik und der Wortbildung - empirisch erschließen. Zu diesem Manifestationstyp seien an dieser Stelle einige Ausprägungsformen exemplifiziert.

Die Lexemwahl kann manchmal - wie im Beleg Nr. (1) - überraschen:

(1) Im Unterrichten konnte er anfangs nicht so gut wie andere Studenten mitkommen, weil Deutsch nicht seine Muttersprache ist. (Nr. 4, S. 5).

Da es sich um einen Studenten handelt, war sicher der Unterricht und nicht das Unterrichten gemeint, also: Im Unterricht konnte er anfangs nicht so gut wie andere Studenten mitkommen, [...]. Vermutlich handelt es sich um eine lexikalische Transferenz, denn das russische Substantiv обучение hat im Deutschen zwei Übersetzungsäquivalente: ,Unterricht‘ und ,Unterrichten'. Möglicherweise diente ein russischer Satz wie В начале обучения он не мог успевать так хоромо, как другие студенты $[\ldots]$ als Prototyp.

Auch viele weitere Ausdrücke folgen russischen Mustern, z. B.

(2) Das war in der Zeit der 90er Jahre (Nr. 3, S. 2).

Die bundesdeutsche Präferenz wäre die Präpositionalphrase in den 90er Jahren gewesen. Der Beleg ist wohl kontaktsprachlich nach dem Vorbild von russ. $в о$ время 90-ых годов entstanden; eine kasachische Transferbasis ist unwahrscheinlich, denn dort heißt es 90-цы (тоқсаныншы) жылдары (wie deutsch: ,in den 90er Jahren').

(3) Insgesamt nahm die Befragung 23 Stunden. (Nr. 4, S. 1)

13 Der Einsatz von ,Kollektion“ als Instrument wird der Konversationsanalyse entnommen (vgl. Deppermann 2014: 22). 
nach russ. опрос занял 23 часа, ${ }^{14}$ im Binnendeutschen würde man eher schreiben: dauerte 23 Stunden oder nahm 23 Stunden in Anspruch.

In ähnlicher Weise dürften die Belege (4) bis (6) auch als proaktive Formtransferenzen des Russischen oder des Kasachischen zu explizieren sein:

(4) So wie ich weiß, haben wir in Deutschland ganze neun Jahre gelebt. (Nr. 3, S. 2).

Der Formulierung so wie ich weiß scheint russ. как я знаю zugrunde zu liegen, die normkonforme Variante wäre soviel ich weiß gewesen.

Proaktive Formtransferenzen ergeben sich auch aus dem unterschiedlichen Bedeutungsumfang oder der semantischen Disparatheit zwischen den Lexemen der deutschen, der russischen bzw. der kasachischen Sprache, z. B.

(5) So leben meine Gedanken zu Kasachstan immer in mir fort. (Nr. 11, S. 6).

Im Deutschland-deutschen Usus hieße es: So leben meine Erinnerungen an $\mathrm{Ka}$ sachstan immer in mir fort. Der Beleg scheint von kasachisch Қазақстан туральы естелік ойлар herzurühren.

Hin und wieder stößt man auf nicht adäquat präfigierte Verben oder bei trennbaren Verben fehlt das Verbpräfix:

(6) Die Teilnahme an den Olympischen Spielen 2018 steht als nächstes großes Ziel der beiden. (Nr. 6, S. 3).

Unmarkiert wäre hingegen die Variante: Die Teilnahme an den Olympischen Spielen 2018 steht als nächstes großes Ziel der beiden an/fest. Hier ist eine Transferenz aus dem Russischen (cmoum) oder aus dem Kasachischen ( $_{\ngtr p}$ ) anzunehmen.

Manche Belege sind durch Labilitäten in der Wortbildung geprägt:

(7) Die Koreaner sind extrem wissensbegierig und lernfreudig, und genau diesen Eindruck habe ich auch hier in Kasachstan. (Nr. 8, S. 4).

Die Form wissensbegierig klingt aus bundesdeutscher Sicht unüblich, vielmehr wäre das Adjektiv wissbegierig als Derivat aus dem Substantiv Wissbegier gängig, bei dem der Wortartwechsel vom Substantiv zum Adjektiv mittels des Derivationsmorphems -ig vorgenommen wurde.

Eine Reihe von Salienzen ist anhand der Kompositionsfuge $-s$ an der Nahtstelle zwischen den unmittelbaren Konstituenten zu beobachten, wie z. B. in der folgenden Überschrift:

(8) Neue Überwachungmethoden (Nr. 7, S. 1).

14 Ein kasachischer Kontakteffekt ist hier auch nicht anzunehmen: Auf Kasachisch würde man 23 сағат уақыт берілді (,nahm 23 Stunden in Anspruch') schreiben. 
Grundsätzlich wird ein Fugenelement zur Verbindung von zwei Konstituenten eines Kompositums eingesetzt; dafür gibt es die Regel, dass eine $s$-Fuge nach Erstgliedern mit dem Suffix -ung zu stehen hat, vgl. Fandrych/Thurmair (2018: 72), also Überwachungsmethoden.

Auch von Kollokationen kann für bundesdeutsche Rezipienten Fremdheit ausgehen:

(9) Jede Familie unserer Schüler wollte ihn zu Gast einladen. (Nr. 20, S. 8).

Womöglich reflektiert der Satz das Muster von kasachisch қонаққа шақыру. Der Standard legt zu sich einladen oder als Gast einladen nahe.

Die Verbindung als gemeinsam irritiert ebenfalls etwas:

(10) Während bei der ersten Art [von Verwaltungsvereinen] durch den Verein internationale Organe geschaffen werden, werden hier staatliche Organe als gemeinsam eingesetzt. (Nr. 9, S. 9).

Dies lässt sich als Transferenzschöpfung nach russ. государственные органы используются как общие interpretieren.

Es finden sich zahlreiche Belege, in denen die verbal-nominale Fügung auffällt, z. B.:

(11) Um den Souveränitätsanspruch der kleineren Staaten zu schonen, [...]. (Nr. 9, S. 9).

Eine unmarkierte Kollokation wäre: dem Souveränitätsanspruch gerecht werden. Der Beleg gilt wohl als Transferprodukt nach russ. [...] чтобы уберечь от притязаний на суверенитет малых государств.

Reine Substantiv-Verb-Verknüpfungen können ebenfalls salient wirken, vgl. Belege (12), (13) und (14):

(12) Das hat mir sozusagen moralische Unterstützung verliehen und ich habe meine mündliche Kommunikation mit großem Erfolg bestanden. (Nr. 1-2, S. 6).

Eine russische Modellgrundlage kann z. B. [...] и сдал устную коммуникацию с большим успехом gewesen sein. Standardtypisch wäre demgegenüber: moralische Unterstützung geben und statt meine mündliche Kommunikation bestanden etwa meine Prüfung „,Mündliche Kommunikation“ mit einer sehr guten Note bestanden.

(13) Das Interesse der Menschen soll in allen Bereichen durch offene Diskussionen geführt werden. (Nr. 9, S. 9).

Die gängige Kollokation wäre das Interesse wecken, also: Das Interesse der Menschen soll in allen Bereichen durch offene Diskussionen geweckt werden. 
(14) Aber nicht einmal den Charakter eines Staatenbundes kann man dem Zollverein beilegen, da [...]. (Nr. 9, S. 9).

Geläufiger wäre allerdings: einer Sache den Charakter (von etw.) beimessen oder zusprechen.

\subsection{2}

Im Bereich der Phraseologie im engeren Sinne und allgemein der figurativen Sprache konnte die datenbezogene Untersuchung ebenfalls aufschlussreiche Befunde generieren. Beispielsweise bietet die DAZ stellenweise Metaphern, die zwar ohne Weiteres verständlich, aber im Deutschland-deutschen Sprachgebrauch eher unüblich sind, z. B.

(15) Die Republik Tadschikistan hat einen vollen Strauß an Umweltproblemen, die oft sehr eng miteinander verbunden sind. (Nr. 12, S. 1).

Man hat hier womöglich mit einer Transferenzübersetzung von russisch полный букет проблем окружающей среды zu tun. Ein bundesdeutscher Journalist hätte sich eher z. B. für ein ganzes Bündel an Umweltproblemen entschieden.

In vielen Fällen mutet die Gebrauchsform der Wendungen ungewöhnlich an:

(16) Es ist ein Gebiet,das [sic!] schon lange im Visier der Terrormiliz „Islamischer Staat" steht. (Nr. 3, S. 4).

Die Struktur im Visier von jmdm. stehen entspricht wohl kaum dem bundesdeutschen Usus, die Nennform des Phraseologismus ist jmdn./etw. im Visier haben. Dementsprechend wäre die sprachüblichere Form: Es ist ein Gebiet, das die Terrormiliz „Islamischer Staat" schon lange im Visier hat. Es ist nicht auszuschließen, dass in die DAZ-Variante auch die russische Wendung находиться под прицелом - obwohl deren Bedeutung nicht ganz identisch ist - unterschwellig hineingespielt hat.

Manche Auffälligkeiten manifestieren sich in der Wahl einer zwar möglichen, aber im binnendeutschen Sprachgebrauch weniger geläufigen Formvariante, z. B.

(17) In Deutschland musste die Familie von Null anfangen. (Nr. 11, S. 6).

Das kontaktsprachliche Muster war offenbar russisch начать с нуля, wobei die Präposition $c$ regulär dem deutschen von entspricht. Die gängigere deutsche Version lautet: bei null anfangen (vgl. Dudenredaktion/Scholze-Stubenrecht 1999: 2764).

In der nächsten Passage kommen sogar mehrere saliente Phraseologismen vor:

(18) Die Magie des neuen Jahres spielt bei mir eine wichtige Rolle. Für mich ist das Neujahr ,ein neues Blatt“ in meinem Leben, das ich mit vollem Wissen über die Vergangenheit und ohne Fehler beginnen will. (Nr. 1-2, S. 6). 
Hier liegen zwei Phraseologietransfers aus dem Russischen vor: Für die Wendung ein nеиеs Blatt (nach russ. новая странииа) würde man im Deutschland-Deutschen ein neues Kapitel und für mit vollem Wissen über etw. (nach russ. с полным осознанием чего-л.) eher in vollem Bewusstsein um etw. schreiben.

Saliente Sprichwörter und festgeprägte Sätze finden sich gelegentlich auch, z. B.

(19) Nicht in die Ferne zu gehen. In der Nähe zu finden. (Nr. 14, S. 5).

In Deutschland würde man sich eher der gängigen Variante des Goethe-Zitats bedienen: Warum in die Ferne schweifen? Sieh, das Gute liegt so nah. ${ }^{15}$

\subsection{3}

In der Grammatik finden sich auch zahlreiche und verschiedenartige Manifestationstypen von Salienz auf sämtlichen Ebenen vom Wort bis zum Text. Beispielsweise in der Morphologie bezüglich der Flexion:

(20) Es ist die sogenannte „Primakov Doktrin“ jener Zeit zu erwähnen, auf deren Basis Russland neben seiner militärischen Präsenz in Zentralasien dem Transit des kasachischen Erdöls und turkmenischen Erdgas durch das Territorium Russlands einen wesentlichen Akzent zu geben begann (Nr. 3, S. 1).

Beim Substantiv Erdgas fehlt die Genitivflexion, also korrekt: Erdgases.

Die Genuszuweisung kann auch diffizil sein:

(21) Im März werden die Wiedergeburt und die Botschaft zusammen eine bilaterale Bildungskonferenz in Astana veranstalten, die sich mit aktuellen Herausforderungen des Schul- und Hochschulbildung in unseren beiden Ländern auseinandersetzt und das Potential der Zusammenarbeit ausloten soll. (Nr. 8, S. 5).

Den kodifizierten Normen nach ist das Substantiv Bildung feminin, sodass das korrekte genitivische Syntagma der Schul- und Hochschulausbildung hieße. Bei diesem Item, wie auch bei mehreren anderen Belegen ist nicht klar zu entscheiden, ob es sich um eine tatsächliche Salienz oder nur um simple Tippfehler etc. handelt.

In anderen Fällen wird nicht normgerecht dekliniert, z. B.

(22) Ich wünsche unserem super Mädels auf jeden Fall viel Erfolg. (6/2017, S. 2).

15 Eine interlinguale Kontaktwirkung kann keine Erklärung liefern, denn die entsprechende Wendung heißt auf Russisch далеко ходить не нужно (,es ist nicht nötig, weit zu gehen') und auf Kasachisch альстан арбалағанша, жақыннан дорбала (,wozu aus der Ferne mit Wagen herantransportieren, lieber aus der Nähe mit Säckchen hierhertragen'). 
Grammatikalisch verlangt die Deklination des Possessivpronomens die plurale Dativform unseren, also unseren super Mädels.

Auch für das nunmehr als veraltet geltende Flexionsallomorph $\{-\mathrm{e}\}$ im Dativ bei Maskulina und Neutra der starken Kasusflexion gibt es Belege, z. B.

(23) im Gegensatze (Nr. 9, S. 9)

oder

(24) dem Vereine (Nr. 9, S. 9). ${ }^{16}$

Im Hinblick auf die Adjektivdeklination finden sich auch Fehloptionen, z. B. Belege (25) und (26):

(25) Es gab viele Schüler, die großes Interesse für das Freiwilliges Soziales Jahr hatten, und unser Gast nahm sich Zeit, um ausführlich auf die Fragen der Schüler einzugehen und Tipps zu geben. (Nr. 8, S. 12).

Eine Verbesserungsvariante wäre: für das Freiwillige Soziale Jahr.

(26) Während die „Karte“ den Forschungsstand abdecken [...] soll, will der Ausstellungsbereich zur Stadt die Menschen in deren direkten Lebensumfeld ansprechen. (Nr. 21, S. 1).

Nach den Demonstrativ- und Relativpronomen dessen und deren wird das nachfolgende attributive Adjektiv immer stark flektiert (vgl. Wöllstein/Dudenredaktion 2016: 962), dementsprechend ist die kanonische Form: [...] in deren direktem Lebensumfeld.

Ein Adjektiv-Adverb-Dilemma ist in Beleg (27) beobachtbar:

(27) Diesjährig wurden bisher 10 Billionen Tenge von der Regierung für die Beseitigung von Schäden aufgebracht. (Nr. 19, S. 1).

Diesjährig ist normalerweise nur als Adjektiv und nicht als Adverb gebräuchlich. Vielleicht geht diese Gebrauchsweise auf das analoge kasachische Adverb биьлл zurück.

Im Bereich der grammatischen Muster der Definitheit treten oft Unsicherheiten auf, was u.U. damit zusammenhängen kann, dass das Russische und das Kasachische artikellose Sprachen sind. Oft wird fälschlicherweise der bestimmte Artikel verwendet:

(28) Die Delegation der deutschen Wirtschaft für Zentralasien (AHK) mit dem Sitz in Almaty unterstützt die deutschen Unternehmen in Kasachstan und anderen zentralasiatischen Staaten. (Nr. 8, S. 8).

16 Interessanterweise wird hingegen in Fällen, in denen das Dativ-e noch durchaus gebräuchlich ist, gelegentlich auf die kürzere Form zurückgegriffen, z. B. in demselben Artikel: im engeren Sinn (Nr. 9, S. 9). 
Hier würde sich eher der Nullartikel anbieten: mit Sitz in Almaty.

Ein umgekehrter Fall liegt bei Beleg (29) vor:

(29) Bei der Entwicklung der rechtlichen Freiheit von Frauen spielt die Frauenbewegung - sogenannte Emanzipation - eine große Rolle. (Nr. 11, S. 10).

Hier wäre statt des Nullartikels ein definiter Artikel die normkonforme Option, also: die sogenannte Emanzipation.

Für das Fehlen eines unbestimmten Artikels soll folgender Beleg stehen:

(30) Ein Ende ist stets Beginn. (Nr. 1-2, S. 2).

Korrekt: Ein Ende ist stets auch ein Beginn.

Im Bereich der Präpositionen findet man in den DAZ-Texten ebenfalls Salienzen, z. B.

(31) Damals hatten wir alle diese karierten Taschen bei der Ausreise. Sie war zu allem gut: Zum Verpacken, aber auch zum darauf schlafen. ${ }^{17}$ (Nr. 52-53. S. 7). ${ }^{18}$

Das könnte eine russische Transferenz sein: для всего хороша. Optimaler wäre auf jeden Fall: Damals hatten wir alle diese karierten Taschen bei der Ausreise. Sie waren für alles gut [...].

Der nächste Beleg deutet ebenfalls auf eine kontaktsprachliche Modellvorlage hin:

(32) Der bayerische Abgeordnete zum Zollparlament war daher nicht nur Abgeordneter Bayerns, [...]. (Nr. 9. S. 9).

Im Deutschland-Deutschen hieße es: Der bayerische Abgeordnete im Zollparlament $[. .$.$] . Die Wahl der Präposition z u$ ist offenbar Folge eines Strukturtransfers aus dem Russischen: denymam кyдa?, also ,Abgeordneter wohin?‘.

Im Bereich der Verben kann z. B. auffallen, dass die Unterscheidung zwischen trennbaren und nicht trennbaren Verben bisweilen diffizil ist:

(33) All das haben die Menschen durchstanden und sind nun auf einem Stand, der heute vorzeigbar ist. (Nr. 17, S. 3).

Das Präfix durch- ist hier trennbar, folglich müsste es heißen: All das haben die Menschen durchgestanden [...].

Dem Rezipienten kann ebenfalls ein nicht-reflexiver Gebrauch reflexiver Verben begegnen:

17 Besser groß und zusammengeschrieben: Daraufschlafen.

18 Obgleich es sich um die Ausgabe 52-53 des Jahrgangs 2016 handelt, wurde sie mit einbezogen, weil sie tatsächlich 2017 erschienen ist. 
(34) In den letzten Jahren haben die Aktivitäten der Frauen in der Zivilgesellschaft fast verdoppelt, besonders bei den Nichtregierungsorganisationen. (Nr. 22, S. 10).

Normkonform wäre sich verdoppelt gewesen.

Auf dem Gebiet der Kasuswahl stößt man auch auf Auffälligkeiten. Im nächsten Satz liegt angesichts der Genitiv-Dativ-Schwankung eine falsche Kasuswahl vor:

(35) Während seiner Auftritte und Gesprächen mit der Öffentlichkeit [...]. (Nr. 9, S. 9).

Die Präposition während regiert den Genitiv, sodass während seiner Auftritte und Gespräche die kanonische Form darstellt.

Bei Beleg (36) fehlt das Vollverb im Nebensatz zur Bildung des mehrteiligen Prädikats, da dieser nur wird als flektiertes temporales Hilfsverb enthält:

(36) Obwohl keiner weiß, wie es in Berlin für mich wird. (Nr. 20, S. 4).

Vollständig wäre der Nebensatz in der Form: [...] wie es für mich in Berlin werden wird. Kontaktsprachlich kann der doppelte Einsatz von werden als Hilfsverb und als Vollverb Schwierigkeiten bereitet haben; im Russischen hieße es einfacher: каково мне будет в Берлине.

Im folgenden Segment tritt eine doppelte Negation auf:

(37) Wir haben keine Feinde in keinem Teil der Erde. Wir bauen freundliche und in allen Parametern auf Vertrauen basierende Beziehungen mit unseren Nachbarn auf. (Nr. 20, S. 1).

Standardadäquat müsste es so heißen: Wir haben in keinem Teil der Erde Feinde. Wir bauen freundschaftliche und in allen Belangen auf Vertrauen basierende Beziehungen mit unseren Nachbarn auf. Vermutlich handelt es sich um eine kontaktsprachliche Musterübernahme aus dem Russischen: У нас нет врагов ни в одной части Земли. Мы строим дружеские и основанные на доверии по всем параметрам отношения с нашими соседями.

Der Tempusgebrauch zeigt oft Auffälligkeiten. Beispielsweise gibt es Belege für den Wechsel der Tempora. In Item (38) variieren Präteritum und Präsens:

(38) Seine letzten Nachfolger sind die Gründer der Dynastie der Großmoguln in Indien, die bis zur britischen Kolonisierung herrschten. Schließlich konvertieren die turko-mongolischen Anführer, die Russland beherrschen - die Goldene Horde-zum Islam. Als Iwan der Schreckliche seine Souveränität zurückerlangte, floh ein Teil dieser Mongolen in Richtung Zentralasien und teilte sich in zwei Zweige auf: [...]. (Nr. 7, S. 5). 
Im folgenden Satz wäre zum klareren Ausdruck des zeitlichen Verhältnisses gleichermaßen eine Revision der Tempusformen anzuraten:

(39) Wenn die Behörden bisher ohne Nennung von Gründen ein Ausreisevisum ablehnen können, sollen Gerichte ,eine Ordnung über das Ausreiseverbot beruhend auf konkreter Gesetzgebung “, [sic!] beschließen. (Nr. 5, S. 1).

Verständlicher wäre wohl - nach Korrektur des Tempus sowie der satzeinleitenden Konjunktion und eines Kommafehlers - z. B. diese Variante: Während die Behörden bisher ohne Nennung von Gründen ein Ausreisevisum ablehnen konnten, sollen nun Gerichte „eine Ordnung über das Ausreiseverbot, beruhend auf konkreter Gesetzgebung “, beschließen.

Die Wahl des temporalen Hilfsverbs kann bei den zusammengesetzten Vergangenheitsformen ebenfalls problematisch sein:

(40) Er kennt alle drei Schulformen, weil er ein Jahr auf der Hauptschule und zwei Jahre auf der Realschule verbracht und anschließend aufs Gymnasium gewechselt ist. (Nr. 4, S. 4).

Da das Verb verbringen seine Perfekt-Form mit dem Hilfsverb haben bildet, müsste es eigentlich heißen: Er kennt alle drei Schulformen, weil er ein Jahr auf der Hauptschule und zwei Jahre auf der Realschule verbracht hat und anschließend aufs Gymnasium gewechselt ist.

Durch Besonderheiten geprägt ist auch der Umgang mit der Syntax in mehrfacher Hinsicht:

(41) Zentralasien wird oft als eine Randregion oder Knotenpunkt zwischen mehreren Kulturen angesehen und weniger bekannt für sein [...]. (Nr. 7, S. 5).

Nach der Ergänzung der fehlenden Satzglieder kann das Segment so lauten: Zentralasien wird oft als eine Randregion oder als ein Knotenpunkt zwischen mehreren Kulturen angesehen und ist weniger bekannt für sein [...].

Nicht selten findet man überflüssige Satzglieder und Attribute, z. B.

(42) Dadurch, dass die Mannschaft jung ist, kann man das nicht immer voraussagen, wer am Ende durchstartet. (Nr. 6, S. 2).

Das hinweisende Wort das ist nicht notwendig: Dadurch, dass die Mannschaft jung ist, kann man nicht immer voraussagen, wer am Ende durchstartet.

Hingegen ist Beleg (43) elliptisch:

(43) Wie herausfinden, ob du zu der Tätigkeit, die du anstrebst, geeignet bist oder noch nicht? (Nr. 14, S. 4). 
Diesen Satz könnte ein russisches Formulierungsmuster inspiriert haben: Кaк догадаться, подходишь ты или нет к деятельности, к которой ты стремишься? Eine grammatikalisch wohlgeformte Formulierung wäre: Wie kannst du herausfinden, $o b$ du für die Tätigkeit, die du anstrebst, schon geeignet bist?

Beleg (44) wirkt etwas verwirrend, da zwei syntaktische Konstruktionen zusammengerutscht sind:

(44) Neuser spielt Trompete und seit fünf Jahren im Quartett, Schlott spielt Saxophon und ist seit 1986 dabei. (Nr. 15, S. 4).

Der Satz wäre auf folgende Weise flüssiger: Neuser spielt Trompete und dies seit fünf Jahren auch im Quartett, Schlott am Saxophon ist (schon) seit 1986 dabei.

Folgende Belege (45) und (46) entsprechen nicht den Stellungsregularitäten:

(45) Sie alle werden nach Kasachstan mit Air Astana fliegen. (Nr. 6, S. 1).

Weder im Russischen noch im Kasachischen ist die Reihenfolge der Objekte fest, sodass hier sowohl ein russischer als auch ein kasachischer Kontaktmechanismus angenommen werden kann. Sprachüblicher wäre: Sie alle werden mit Air Astana nach Kasachstan fliegen.

(46) Das Projekt legt die fünf wichtigen Richtlinien und Schwerpunkte fest über die Weiterentwicklung Usbekistans von 2017 bis 2021. (Nr. 9, S. 9).

Beleg (46) verkörpert einen Hauptsatz, der mit einem mehrteiligen Prädikat operiert. Im Vorfeld befindet sich das Subjekt das Projekt, während die linke Satzklammer mit dem finiten Verb legt und die rechte Satzklammer mit der trennbaren Verbpartikel fest besetzt ist. Die Verbklammer umschließt das mit dem Akkusativobjekt die fünf wichtigen Richtlinien und Schwerpunkte belegte Mittelfeld, das Nachfeld wird vom Präpositionalobjekt über [besser: für] die Weiterentwicklung Usbekistans von 2017 bis 2021 gebildet. Da Objekte grundsätzlich ins Mittelfeld einzugliedern sind, könnte die unmarkierte Satztopologie so aussehen: Das Projekt legt die fünf wichtigen Richtlinien und Schwerpunkte für die Weiterentwicklung Usbekistans von 2017 bis 2021 fest.

In Bezug auf die Textbildung bzw. -gestaltung ist die hohe Frequenz unverbundener Strukturen anzusprechen:

(47) Das Land importiert Maschinen, elektrische Ausrüstungen, chemische Produkte, Autos und Autoteile, pharmazeutische Erzeugnisse aus Deutschland. (Nr. 8, S. 8).

Zwischen den Elementen Autoteile und pharmazeutische Erzeugnisse wäre statt des Kommas der koordinierende Konjunktor sowie angebrachter. 


\subsection{4}

Bekanntlich spiegeln kommunikative Produkte das gegebene soziokulturelle Setting mit den entsprechenden Narrativen wider, ist doch jeder Sprachträger Teil seiner eigenen Diskurskultur und bringt die entsprechenden kommunikativen Erfahrungsstrukturen und Wissensbestände in Textstrukturierungs- und Formulierungsroutinen hervor (vgl. Földes 2018b: 136). Beispielsweise hat bereits Heusinger (1995: 7) betont: „Textsortenwissen beruht auf überlieferter Alltagserfahrung aus unzähligen Akten sprachlich-kommunikativer Tätigkeit, das heißt, es ist im Alltagsbewußtsein verankert“. Neuerdings wird Kulturalität sogar als ein Textualitätsmerkmal angesehen (vgl. Zhao 2018).

In diesem Sinne weisen DAZ-Beiträge im gegebenen sprach- und kommunikationskulturellen Rahmen als Katalysator spezifische Eigenheiten auf. In Bezug auf die Stilgestaltung ist als Globalbewertung z. B. ein gewisser Eklektizismus zu konstatieren. Im Einzelnen lassen sich diverse Merkmale herausarbeiten.

Von der Wortsemantik bzw. Wortstilistik her sei folgender Beleg genannt:

(48) „Der Familienzusammenhalt unter den Russlanddeutschen ist sehr starkes ist eine gemeinsame Wanderung über Jahrhunderte, die sie zusammenschweißt", sagt Thiessen. (Nr. 52-53, S. 7).

Angesichts des leidgeprüften Schicksals der Russlanddeutschen würden sich eher Weg oder Reise anbieten, da man mit Wanderung vielmehr etwas Gemütliches assoziiert.

Salient ist die häufige Verwendung umgangssprachlicher Wörter, Ausdrücke und grammatischer Phänomene auch in stilistisch-pragmatisch gehobeneren Textsorten, z. B. die Lexemvariante alleine $^{19}$ (statt allein), vgl. Dudenredaktion/Scholze-Stubenrecht (1999: 166).

(49) Trotzdem er unschuldig war, wurde er zum Tod durch Erschießen verurteilt. (Nr. 9, S. 4).

Die Verwendung von trotzdem als konzessive Konjunktion ist umgangssprachlich oder regional (vgl. Dudenredaktion/Scholze-Stubenrecht 1999: 3983 und Wöllstein/ Dudenredaktion 2016: 596), in einer informierenden Textsorte wie Bericht wären eher folgende Versionen zu erwarten: Trotz der Tatsache, dass er unschuldig war, [...] oder Obwohl er unschuldig war, [...].

Wortwiederholungen ${ }^{20}$ verschiedenen Typs kommen nicht selten vor, z. B. in einem Lead zweimal nach Deutschland:

19 In mehreren Texten (z. B. Nr. 20, S. 4).

20 Neben Wortwiederholungen gibt es auch Wiederholungen grammatischer Strukturen: Innerhalb einer kurzen Randspalte (Nr. 21, S. 1) kommt in Finalsätzen die Infinitivgruppe mit um...zu dreimal vor (und die Verben profitieren und umsetzen je zweimal). 
(50) [...] deshalb entschied sich mein Opa mit ihr nach Deutschland zu gehen, um ihr eine gute Ausbildung zu ermöglichen. Im Dezember 1992 ist sie nach Deutschland gekommen [...] (Nr. 3, S. 2).

Noch eklatanter sind Fälle, in denen Wortwiederholungen in ein und demselben Satz zu beobachten sind, wie:

(51) In dem sehr abwechslungsreichen Programm standen Ausflugsziele rund um Astana auf dem Programm. (16/2017, S. 7).

Zwecks stilistischer Aufbesserung des Satzes könnte das Programm in der rechten Satzklammer wie folgt ersetzt werden: In dem sehr abwechslungsreichen Programm waren Ausflugsziele rund um Astana geboten.

Den Stil betreffend fällt in Item (52) die zweifache Verwendung desselben Wortes auf:

(52) Mit bunten Luftballons wurden die Schülerinnen und Schüler aus Deutschland bereits am Flughafen sehr herzlich von ihren Gastfamilien empfangen. [...] Die deutsch-kasachische Schülergruppe erlebte bei ihren Exkursionen und Unternehmungen unvergessliche Momente und es entstanden dabei herzliche Freundschaften. (Nr. 16, S. 7).

Eine Optimierungsoption wäre, das zweite herzlich durch enge zu ersetzen (also enge Freundschaften). Das Adjektiv herzlich unterlag wohl einem Transfer semantischer Art von russ. сердечная (дружба).

DAZ-Texte bedienen sich oft multipler Genitivattribute, die wahrscheinlich auf ein russisches oder kasachisches Konstruktionsmuster zurückgehen, im Deutschen jedoch schwer verständlich sind und daher eine ungewöhnliche Ausdrucksweise ergeben, vgl. den dreifachen Genitiv anhand der Namensgebung der Bestandteile der Schau in Beleg (53):

(53) Dass an diesem Image festgehalten werden soll, erfährt man schon anhand der Namensgebung der Bestandteile der Schau: „Karte der Zukunft" und „Stadt der Zukunft“. (Nr. 21, S. 1).

Aus linguistischer Sicht verdient zudem das Kompositum Namensgebung ${ }^{21}$ eine Anmerkung, da der entsprechende Terminus ohne Fugen-s Namenforschung heißt.

Ein Eklektizismus ergibt sich schließlich aus dem Nebeneinander eher alltagssprachlich-unprofessioneller Formulierungspraktika einerseits und gehobener bzw. bildungssprachlicher Sprachmuster andererseits. Im letzteren Zusammenhang sei Beleg (54) angeführt, der im zweiten Satz mit einer gleichsam poetischen Ellipse operiert:

21 Ähnlich auch Namensgeber (Nr. 17, S. 10). 
(54) Flucht aus dem Heimatland [...] vom Kindheitsraum bis hin zum gefragten deutschen Journalisten - das sind die Lebensphasen von Krsto Lazarević. Seine Geschichte. (Nr. 4, S. 4).

Was die Texte als Ganzes anbelangt, entsteht der Gesamtendruck, dass manche Artikel wenig lebendig, eher sehr trocken gehalten sind, z. B. der Artikel „Russlands Handel mit Zentralasien“ (Nr. 3, S. 1 und 4). In einer bundesdeutschen Zeitung, z. B. in der Süddeutschen, würde hingegen ein Artikel auch im Wirtschaftsteil mit einem interessanten Aufhänger beginnen, sodass die Texte auch abseits von Zahlen ansprechend sind. Dagegen beginnt der genannte DAZ-Artikel, wie auch viele andere, mit einem einfallslos formulierten Vorspann narrativen Charakters.

Ein Teil der Artikel erinnern noch an die langweilig-umständliche bürokratische Formulierungspraxis der sowjetischen Zeit:

(55) Die deutsche Parlamentarische Freundschaftsgruppe zu Besuch in Kasachstan. [...]. „Es freut mich, dass es im deutschen Bundestag eine parlamentarische Freundschaftsgruppe gibt, die sich über Jahrzehnte gegenüber den deutschen Regierenden und dem Bundestag, aber auch hier am Parlament für diese Freundschaft einsetzt [...]". (Nr. 17, S. 1). ${ }^{22}$

\subsection{5}

Auf der Ebene der Grafie können ebenfalls Besonderheiten registriert werden, beispielsweise gibt es relativ viele Fehler in der Worttrennung am Zeilenende, z. B.

(56) Rus-sland (Nr. 3, S. 1, 3, 4 und 52-53, S. 7). ${ }^{23}$

In Bezug auf die Neuregelung der deutschen Rechtschreibung findet man Unsicherheiten hinsichtlich ihrer Umsetzung. Ein Beispiel zur Regelgruppe Groß- und Kleinschreibung liefert Beleg (57):

(57) Eine unerbittliche Notwendigkeit stellte nach jeder Krise die Grenzen des alten Zollvereins wieder her; kalte politische Köpfe konnten mit mathematischer Sicherheit den Verlauf des Streites im voraus berechnen. (Nr. 11, S. 2).

Seit der Orthografiereform von 1996 ist die richtige Schreibung: im Voraus.

Ein umgekehrtes Beispiel, also eine Hyperkorrektion im Sinne von Übergeneralisierung, stellt Beleg (58) dar:

22 Im Anschluss wird noch zweimal die Bezeichnung „deutsch-Zentralasiatische Parlamentariergruppe“ bzw. die ,deutsch-zentralasiatische[n] Parlamentariergruppe“ (mit jeweils unterschiedlicher Rechtschreibung) genannt.

23 Auf eine Aufzählung von Belegen für Inkonsequenzen und Fehler verschiedener Art (z. B. in der Getrennt- und Zusammenschreibung) wird aus Umfangsgründen verzichtet. 
(58) Alles in Allem aber war es eine wunderschöne Reise, bei der wir Unglaubliches gesehen und neue Leute kennengelernt haben. (Nr. 11, S. 8).

Den geltenden Rechtschreibvorschriften würde alles in allem entsprechen. ${ }^{24}$

Auch zur Interpunktion können Besonderheiten benannt werden. Beispielsweise wird in Beleg (59) der Anfang des Einschubs mit Gedankenstrich ${ }^{25}$ und das Ende mit Komma gekennzeichnet:

(59) Als Teil der Bewegung gilt auch die Frauendemonstration in Russland mit dem Motto „Brot und Frieden“ im Jahr 1917 am 8. März - nach dem alten russischen Kalender am 23. Februar, die die Februarrevolution auslöste. (Nr. 11, S. 10).

Außerdem ist hinzuzufügen, dass die Aussage inhaltlich unterkomplex und sehr gewagt ist, denn es gehörte doch mehr zu den „Auslösern“ der Revolution.

Hin und wieder überraschen in der bundesdeutschen Mediensprache unübliche Schreibweisen, z. B. im folgenden Beleg zum „Kniffel-Schach“:

(60) Schwarz am Zug setzt patt mit 1 Zug. (Nr. 13, S. 12 und Nr. 15, S. 12).

Eine bundesdeutsche Zeitung hätte bestimmt nicht die Ziffer-Variante, sondern die ausgeschriebene Form mit einem Zug gewählt.

Die Transkriptionspraxis weist Uneinheitlichkeit auf, vgl. beispielsweise Beleg (61), in dem das russische Substantiv привет im Titel als Priwet, im Fließtext hingegen als Privet wiedergegeben wird.

\subsection{6}

Einen besonderen Typ von Salienzen verkörpern mehrsprachige und hybride Sprachpraktiken. Ab und zu begegnen dem Leser sogar dreisprachige Ensembles wie in der Überschrift eines Berichts über Kasachstan Priwet! Rachmet und auf Wiedersehen! (Nr. 8, S. 9), wobei russ. privet ,Gruß, Grüß dich!' und kasachisch rachmet ,danke‘ bedeutet. Analog heißt der letzte Satz des Artikels:

(61) „Rachmet“, rollt es über meine Zunge, worauf er sich lächelnd mit einem „Privet! Rachmet und auf Wiedersehen!", verabschiedet. (Nr. 8, S. 9).

24 Innerhalb desselben Artikels kommt allerdings auch die normative Schreibvariante vor (Nr. 11, S. 9).

25 Für diesen Artikel ist eine Häufung von Gedankenstrichen, jedoch in unterschiedlicher Funktion bzw. Verwendung charakteristisch. 


\subsection{7}

Salienzen treten oft auf der Ebene der Ausdrucksgestaltung auf:

(62) Etwa 90\% des deutschen Handelsvolumens in Zentralasien und ca. 75\% des deutschen Exports nach Zentralasien entfallen auf den Anteil Kasachstans. (Nr. 8, S. 8).

Korrekter wäre: [...] entfallen auf Kasachstan.

In demselben Artikel heißt es:

(63) Das stellt die EU in die Position des größten Handelspartners des Landes im Außenhandel. (Nr. 8, S. 8).

Üblicher wäre statt der Nominalgruppe Handelspartner im Ausland einfach das Kompositum Außenhandelspartner oder eventuell Partner im Außenhandel.

Ebenda findet man:

(64) In der langen Perspektive kann Kasachstan auch Gas nach Deutschland exportieren, wenn Kasachstan und Turkmenistan das Trans-Caspian Crude Oil Export System anschließen können. (Nr. 8, S. 8).

Hier würde sich bei der satzinitialen Präpositionalphrase eher in langfristiger Perspektive oder einfach nur langfristig anbieten.

Die Formulierung in Item (65) scheint der Ausdrucksstruktur des Russischen zu folgen:

(65) Schicken Sie die ausgefüllte Bewerbung bitte bis zum 14. Juni 2017 an E-Mail: [...]. (Nr. 21, S. 12).

Die Formulierung am Sequenzende scheint als Modell-Transferenz auf Russisch на е-mail zurückzugehen. Ein bundesdeutsches Printmedium würde eher schreiben: Schicken Sie die ausgefüllte Bewerbung bitte bis zum 14. Juni 2017 an die folgende E-Mail-Adresse: [...].

In Beleg (66) liegt - neben grammatischen Unebenheiten - in dem Klammereinschub eine Kontamination vor:

(66) Die zweite Emanzipation (ab 1960er) zielte auf einen gesellschaftlichen Wertewandel $a b,[\ldots]$. (Nr. 11, S. 10).

Entweder wäre $a b$ 1960, $a b$ den 1960er Jahren oder eben ab den Sechzigerjahren des 20. Jahrhunderts korrekt.

Die Zwischenüberschrift Nr. (67) fällt im Bereich der Verbalphrase auf:

(67) Zusammenarbeit zwischen Kasachstan und Deutschland erstarkt und wird ausgebaut. (Nr. 3, S. 8). 
Optimaler wäre: Zusammenarbeit zwischen Kasachstan und Deutschland wird gestärkt und ausgebaut.

\subsection{8}

Viele Texte enthalten eindeutige sprachformbezogene Fehler verschiedener Art, die wohl aus Unachtsamkeit passiert sind, vgl. Beleg (68):

(68) Nachdem diplomatische Beziehungen zwischen der Bundesrepublik Deutschland und Kasachstan am 11. Februar 1992 aufgenommen hatten, begannen beide Länder wirtschaftlich bilateral und multilateral zu kooperieren. (Nr. 8, S. 8).

Neben der Korrektur des Satzsubjekts bedarf der Umgang mit den Staatsbezeichnungen im Sinne der Gleichbehandlung einer Vereinheitlichung: Entweder sollten bei den beiden die Kurzformen (Deutschland bzw. Kasachstan) oder die Langformen (Bundesrepublik Deutschland und Republik Kasachstan) verwendet werden. Außerdem wirken drei Fremdwörter nebeneinander (bilateral, multilateral und kooperieren) unschön. Daher wäre eine korrektere Option: Nachdem Deutschland und Kasachstan am 11. Februar 1992 diplomatische Beziehungen aufgenommen hatten, begannen beide Länder, wirtschaftlich bi-und multilateral zusammenzuarbeiten.

\subsection{9}

In Bezug auf die Typografie sei exemplarisch folgender Befund genannt: der fehlende Wortzwischenraum zwischen dem Punkt und dem nachfolgenden Wort: ${ }^{26}$

(69) Gesang zum 8.März. (Nr. 11, S. 10).

\subsubsection{0}

Hinsichtlich der Kontextualisierung von raumbezogenen Salienzen operiert die DAZ mit verschiedenen kommunikativen Strategien. Beispielsweise wird das inhaltlich substanzielle Schlüsselwort Zentralasien (bzw. zentralasiatisch) öfter abgekürzt.

(70) $Z A$ (Nr. 3, S. 1). ${ }^{27}$

Dieses nicht aufgelöste Akronym ist für Außenseiter nicht ohne weiteres verständlich. Auch viele weitere, vor allem kasachstanspezifische, Kurzwörter erscheinen für bundesdeutsche Rezipient(inn)en ohne erläuternde bzw. paraphrasierende Kommentare als unbekannt und intransparent, z. B.

(71) KAZGUU (Nr. 15/2017, S. 1).

26 Einen ähnlichen Fehler enthält auch Beleg (16).

27 Für zentralasiatisch. 
Hinter dem Buchstabenwort verbirgt sich die Kasachische Universität für Geistesund Rechtswissenschaften; auf Kasachisch: Қазақ гуманитарлық заң университеті und auf Russisch: Казахский гуманитарно-юридический университет (КазГЮУ) in Astana.

Es gibt auch inverse Fälle, in denen Wörter und Ausdrücke erläutert werden, die in Deutschland keiner Erläuterung bedürfen, wie z. B. in Beleg (72) beim Kompositum Grasschollen, dessen Bedeutung eine bundesdeutsche Zeitung vermutlich nicht erklären würde.

(72) Bereits verdorrte Grasschollen, das sind rechteckige Rollrasenelemente, wirken liegengelassen. (Nr. 21, S. 1).

Zum Schluss eine Auffälligkeit, die weder sprachlicher noch kultureller, sondern redaktioneller Natur ist:

(73) Aber in den Jahren 1997-2000 lag der Import bei mehr als 8\%, während der Import unter 4\% lag. (Nr. 3, S. 4)

- eines davon muss Export sein.

\subsubsection{1}

Dass in der DAZ eine Häufung diverser Auffälligkeiten verschiedener Art auf kleinstem Raum auftreten kann, soll abschließend folgender Beleg illustrieren:

(74) Die Stadt Almaty hat sich akribisch vorbereitet: Schüler und Studenten wurden freigestellt und somit viele Tausende Freiwilliger gewonnen, der Neujahrsschmuck erleuchtet die Hauptstraßen, große Plakate sollen den urbanen Raum verschönen und eine hohe Polizeipräsenz die Sicherheit gewährleisten, auch freilaufender Tiere wurde sich entledigt. Tatsächlich wurde sich auf das Event über die Maßen vorbereitet. Fast scheint es, als soll bewiesen werden, dass auch eine Winterolympiade drin gewesen wäre. (Nr. 6, S. 1).

Hier kann man mindestens acht Details optimieren: Im Satzvorfeld ist die Gattungsbezeichnung Die Stadt nicht notwendig, denn besonders in Kasachstan weiß doch jeder, dass Almaty eine Stadt ist, vielmehr könnte durch diese Artikelphrase die eher offiziös-fachsprachlich anmutende Nominalphrase den urbanen Raum ersetzt werden. Statt verschönen sollte man eher verschönern schreiben. Da nach große Plakate das Modalverb sollen im Plural steht, ist es nach Polizeipräsenz im Singular noch einmal zu setzen. Die Reflexivpassive, also die unpersönlichen reflexiven Passivbildungen reflexiver Verben, sind ebenfalls nicht unproblematisch (vgl. Wöllstein/Dudenredaktion 2016: 560), nämlich wurde sich entledigt und wurde sich vorbereitet. Die Präpositionalphrase über die Maßen klingt ungewöhnlich, vermutlich ist dieser Ausdruck 
kontaktsprachlich durch kasachisch шамадан тыс (,über die Maße') oder eventuell durch das russische Adverb чрезмерно $=$,übermäßig' (через $=$,über ${ }^{\star}+$ мера $=, \mathrm{Ma}^{\circ}$ ) motiviert. Im letzten Satz wäre statt der Indikativform soll das konjunktivische solle die bessere Variante und das Präpositionaladverb drin wirkt an dieser Stelle wegen dessen gesprochen-sprachlicher Prägung (vgl. Wöllstein/Dudenredaktion 2016: 592) eher umgangssprachlich und passt innerhalb des berichtenden Kontexts nicht wirklich. Folglich könnte eine unmarkierte Formulierung so lauten: Almaty hat sich akribisch vorbereitet: Schüler und Studenten wurden freigestellt und somit viele Tausende Freiwilliger gewonnen, der Neujahrsschmuck erleuchtet die Hauptstraßen, große Plakate sollen die Stadt verschönern und eine hohe Polizeipräsenz soll die Sicherheit gewährleisten, auch freilaufende Tiere wurden eingefangen. Tatsächlich hat man für dieses Ereignis alles beinahe schon pedantisch organisiert. Fast scheint es, als solle bewiesen werden, dass auch eine Winterolympiade hätte stattfinden können.

\section{3}

Der andere paradigmatische Großbereich, der von Salienzen primär kulturbezogener Provenienz, soll demnächst den Gegenstand eines anderen Aufsatzes bilden (Földes, im Druck), sodass an dieser Stelle lediglich ein exemplarisches Beispiel angeführt sei. So ist in Item (75) das multisegmentale Kurzwort anzusprechen:

(75) Wie geplant war, traf der Bundeskanzler der BRD, Roman Herzog, zu einem dreitägigen offiziellen Besuch in Almaty ein. (Nr. 3, S. 8). ${ }^{28}$

In Deutschland wird das Akronym $B R D$ in offiziellen Kontexten - da es u.U. als kontroverser Begriff betrachtet wird (vgl. Hahn 1995: 317ff.) - nicht verwendet, man würde eher schreiben: der deutsche Bundeskanzler oder eventuell der Bundeskanzler der Bundesrepublik Deutschland. ${ }^{29}$

\section{AUSWERTUNG UND SCHLUSSFOLGERUNGEN}

Anhand der evidenzbasierten sprachbezogenen - jedoch bezüglich des Korpusumfangs begrenzten - Analyse sind verschiedene charakteristische Merkmale im Hinblick auf die Sprachlichkeit der DAZ deutlich geworden. Allgemein konnte für die untersuchte schriftkulturelle Praxis eine besondere Form von Mehrschriftlichkeit festgestellt werden. Terminologisch wird hier unter Mehrschriftlichkeit (vgl. Riehl 2018: 188) im Sinne von multiliteracy die Beherrschung mehrerer Sprachen im schriftsprachlichen Ausdruck verstanden, im Gegensatz zu Mehrschriftigkeit (vgl. Erfurt 2017: 24), die im Sinne von Biliteralismus, wenn es um unterschiedliche Schriftsysteme geht, den

28 Es gibt eine Anzahl weiterer Vorkommensbelege, z. B. Nr. 8, S. 16; Nr. 10, S. 1; Nr. 12, S. 1; Nr. 15, S. 1; Nr. 16, S. 1; Nr. 19, S. 1 und Nr. 20, S. 1.

29 Auf ein anderes Blatt gehört der sachliche Irrtum, dass Roman Herzog Bundespräsident, nicht Bundeskanzler war. 
Sonderfall bezeichnet. Wichtig ist dabei die Frage, ob man es im Kontext kasachstandeutscher Texte mit mehrfacher Literalität oder lediglich - wie Maas (2008: 478) formuliert - mit einem literaten Ausbau in unterschiedlichen skribalen Formen zu tun hat. Es ist davon auszugehen, dass letzten Endes inter- bzw. genauer: transkulturelle Texte $^{30}$ vorliegen. Somit geht es um eine hochgradig komplexe sprachlich-kulturelle Basiskonfiguration, als deren Folge der DAZ der Status einer mehrfach kulturasymmetrischen Grenzgänger-Zeitung - einer Species sui generis - zuzuschreiben ist. Erstens, weil sie im Wirkungsraum zwischen Deutsch, Russisch und Kasachisch funktioniert, zweitens, weil eine besondere Asymmetrie feststellbar ist: Das Formmaterial der Sprache ist grundsätzlich deutsch, während Textkonzept und Formulierungsmuster infolge des kulturellen Referenzrahmens russischer und kasachischer Provenienz sind. Deutschsprachige Textproduktion im kasachisch und russisch geprägten soziokulturellen Impulsareal stellt mithin einen besonderen Medientyp und folglich eine besondere Ausprägung der Medienkommunikation dar.

Die Auseinandersetzung mit der Beleglage hat gezeigt, dass die einzelnen Textprodukte sowohl in der sprachlichen Gestaltung wie auch im journalistischen Anspruch zum Teil stark divergieren, was möglicherweise durch die sprach- und kulturheterogene Zusammensetzung des Redaktionsteams bedingt ist (vgl. zur Problematik Földes 2018b: 143). Uneinheitlichkeiten treten selbst innerhalb ein und desselben Textes auf: Sie äußern sich mitunter in einer scheinbar unmotivierten Koexistenz im Prinzip inkompatibler Einheiten. Außerdem hat die Untersuchung interessante Wechselwirkungen von Schriftsprachkompetenzen und Formen des Transfers zutage gebracht, vgl. z. B. in Abschnitt 4.2.4. Es konnte eruiert werden, dass zu den wichtigsten Profilelementen der DAZ-Sprachlichkeit vor allem (virulente oder latente) Phänomene von Kultur- und Sprachkontakten gehören. Einige dieser Besonderheiten werden vermutlich bewusst eingesetzt oder von der Redaktion zumindest in Kauf genommen, denn sie üben u.U. auch eine Signalfunktion aus, indem sie das pragmatische Potenzial des DAZ-Textkosmos erhöhen und ihr eine authentische kasachstanische Färbung geben können, vgl. Földes (2018b: 144). Größtenteils scheint es sich allerdings um eine nicht intendierte Folge der mehrsprachigen und inter- bzw. transkulturellen Umwelt bzw. Lebensgestaltung zu handeln.

In summa ist festzustellen, dass die DAZ dank ihrer spezifischen Sprachlichkeit (und Kulturalität) ein bemerkenswertes Druckmedium darstellt. Die Studie hat ein Spektrum an aufschlussreichen Befunden in sämtlichen Dimensionen des untersuchten Schriftsprachengebrauchs ergeben. So war - neben den erwähnten unmittelbaren Mehrsprachigkeits- und Kontaktmanifestationen - eine geringe Registerdifferenzierung und dadurch oft eine gewisse Uneinheitlichkeit, also Heterogenität und Schwankungen in stilistisch-pragmatischer Hinsicht und/oder in der Satz- und Textkonstruktion, ${ }^{31} \mathrm{zu}$

30 Burka (2016: 35ff.) reflektiert ausführlich über Inhaltsstruktur und Verwendungspotenzial des Begriffs ,interkulturelle Literatur`.

31 Diese lassen sich - in der Terminologie von Pörksen/Detel (2012: 235) - als Kontextverletzungen charakterisieren. 
beobachten. ${ }^{32}$ Aus diesen intra- und z.T. interlingualen hybriden Verhältnissen resultiert mitunter eine Differenzspannung. Es lässt sich schlussfolgern, dass die eruierten Verwendungsbesonderheiten aus drei Prozessen typologisch unterschiedlicher Art resultieren (vgl. Földes 2018a: 74): (1) aus prototypischen Sprachkontaktmechanismen, also aus Transfers oder Nachahmungen von Elementen, Strukturen und Modellen der Kontaktsprachen Russisch und Kasachisch, vgl. z. B. Belege Nr. (3) und (9); (2) aus Verfremdungsprozessen, z. B. aus Kontrastverschiebungen oder -übertreibungen, die sich aus einer unsicheren Beherrschung der Zielsprache Deutsch ergeben, etwa durch Übergeneralisierung des Sprachsystems, ein Beispiel für diesen Phänomentyp könnte Beleg Nr. (26) und (33) sein; (3) aus Normverletzungen aufgrund von Unachtsamkeit, d. h. Flüchtigkeitsfehler bzw. problematische oder grenzwertige Formulierungen, die selbst bei Textproduzenten mit exzellenter (nativer) Sprachbeherrschung vorkommen, z. B. Beleg Nr. (50) und (69). Quantitativ überwiegen die mehrsprachigkeits-, kontaktund/oder kompetenzbedingten Auffälligkeiten, wobei sich die sprachliche Strahlkraft des Russischen als größer erwies als die des Kasachischen. Zu einer wirklich sachangemessenen Interpretation der Befunde müsste man allerdings wissen, (a) welchen sprachbiografischen Hintergrund der eigentliche Verfasser hat, (b) ob der gegebene Zeitungstext vielleicht ein Übersetzungstext ist und wer in diesem Fall der Übersetzer war und (c) wer mit welchen sprachlichen Kompetenzbeständen und mit welcher redaktionellen Leitkonzeption die Texte korrigiert und redigiert hat (vgl. Földes 2018a: 74), letztlich hat man es doch mit kollaborativen Texten zu tun. Es wäre zudem aufschlussreich, herauszufinden, ob es bei der DAZ (und im globalen Maßstab allgemein) besondere - „naive“ oder elaborierte - journalistische Leitmodelle interkultureller Textproduktion für auslandsdeutsche Medien gibt.

\section{AUSBLICK}

Schmitz (2016: 904) konstatiert: „Medienlinguistische Forschungen erweitern die hergebrachten Perspektiven konventioneller Sprachwissenschaft". Diesem Postulat ist zuzustimmen und angesichts der obigen Ausführungen kann man sogar dezidierter formulieren, nämlich, dass die Beschäftigung mit dem auslandsdeutschen Pressediskurs noch zusätzliche u. a. spezifische interkulturell-linguistische Einsichten ermöglicht.

\section{Literatur}

AGDM (2018) Arbeitsgemeinschaft Deutscher Minderheiten in der FUEN: Deutsche Minderheit in Kasachstan. In der FUEN vertreten seit 1993. https://agdm.fuen.org/ mx5-mitglied-51/kazakhstan/ [30.11.2018].

BURKA, Bianka (2016) Manifestationen der Mehrsprachigkeit und Ausdrucksformen des ,Fremden 'in deutschsprachigen literarischen Texten: exemplifiziert am Beispiel von Terézia Moras Werken. Tübingen: Narr Francke Attempto.

DAZ (2018) Die Zukunft der kasachstandeutschen Medien. http://daz.asia/blog/die-zukunft-der-kasachstandeutschen-medien/ [30.11.2018].

32 Ähnliches wurde über die Sprache der „Moskauer Deutschen Zeitung“ in der Studie von Földes (2018a: 73) ebenfalls herausgearbeitet. 
DEPPERMANN, Arnulf(2014) „Konversationsanalyse: Elementare Interaktionsstrukturen am Beispiel der Bundespressekonferenz." In: S. Staffeldt/J. Hagemann (Hrsg.), Pragmatiktheorien: Analysen im Vergleich. Tübingen: Stauffenburg, 19-47.

DUDENREDAKTION/Werner SCHOLZE-STUBENRECHT (Hrsg.) (1999) Duden. Das große Wörterbuch der deutschen Sprache in zehn Bänden. 3., völlig neu bearb. u. erw. Aufl. Mannheim/Leipzig/Wien/Zürich: Dudenverlag.

ERFURT, Jürgen (2017) „Von der Mehrsprachigkeit zur Mehrschriftigkeit. Elemente einer Theorie des sprachlichen Ausbaus." Bulletin VALS-ASLA, Numéro spécial, $t$. $1,11-38$.

FANDRYCH, Christian/Maria THURMAIR (2018) Grammatik im Fach Deutsch als Fremd- und Zweitsprache. Grundlagen und Vermittlung. Berlin: Erich Schmidt.

FÖLDES, Csaba (2018a) „Mediensprache im Kontakt der Kulturen: Beispiel ,Moskauer Deutsche Zeitung، “ 'In: C. Földes (Hrsg.), Sprach- und Textkulturen - interkulturelle und vergleichende Konzepte. Tübingen: Narr Francke Attempto, 43-77.

FÖLDES, Csaba (2018b) „Xenismen in der auslandsdeutschen Pressesprache. Reflexionen anhand der Moskauer Deutschen Zeitung." Jahrbuch des Bundesinstituts für Kultur und Geschichte der Deutschen im östlichen Europa 26: Sprache, 121-144.

FÖLDES, Csaba (Im Druck) „Mediendiskurs in kulturell mehrfach kodierten Räumen: Betrachtungen über die ,Deutsche Allgemeine Zeitung in Kasachstan." In: C. Földes (Hrsg.), Kontaktvarietäten des Deutschen im Ausland. Tübingen: Narr Francke Attempto.

HAHN, Silke (1995) „Vom zerrissenen Deutschland zur vereinigten Republik. Zur Sprachgeschichte der, deutschen Frage“." In: G. Stötzel/M. Wengeler (Hrsg.), Kontroverse Begriffe. Geschichte des öffentlichen Sprachgebrauchs in der Bundesrepublik Deutschland. Berlin/New York: De Gruyter, 285-353.

HEUSINGER, Siegfried (1995) „Textsorten in der interkulturellen Kommunikation ein Problemaufriß.“" Linguistica 35/1, 7-20.

LENZ, Alexandra N. (2010) „Zum Salienzbegriff und zum Nachweis salienter Merkmale.“ In: Chr. A. Anders/M. Hundt/A. Lasch (Hrsg.), Perceptual dialectology. Neue Wege der Dialektologie. Berlin/New York: De Gruyter, 89-110.

LINK, Jürgen (1998) „Literaturanalyse als Interdiskursanalyse. Am Beispiel des Ursprungs literarischer Symbolik in der Kollektivsymbolik. “In: J. Fohrmann/H. Müller (Hrsg.), Diskurstheorien und Literaturwissenschaft. Frankfurt a. Main: Suhrkamp, 284-307.

MAAS, Utz (2008) Sprache und Sprachen in der Migrationsgesellschaft: Die schriftkulturelle Dimension. Göttingen: V \& R Unipress/Universitätsverlag Osnabrück.

PARKINSON, Brian (2014) „Soziale Wahrnehmung und Attribution.“ In: K. Jonas/W. Stroebe/M. Hewstone (Hrsg.), Sozialpsychologie. 6., vollst. überarb. Aufl. Berlin/ Heidelberg: Springer, 65-106.

PÖRKSEN, Bernhard/Hanne DETEL (2012) Der entfesselte Skandal: Das Ende der Kontrolle im digitalen Zeitalter. Köln: von Halem.

PURSCHKE, Christoph (2011) Regionalsprache und Hörerurteil: Grundzüge einer perzeptiven Variationslinguistik. Stuttgart: Steiner. 
RÁCZ, Péter (2013) Salience in sociolinguistics: A quantitative approach. Berlin/Boston, Mass.: De Gruyter Mouton.

RIEHL, Claudia Maria (2018) „Mehrschriftlichkeit und Transfer.“ In: J. Roche/E. Terrasi-Haufe (Hrsg.), Mehrsprachigkeit und Spracherwerb. Tübingen: Narr Francke Attempto, 187-198.

SCHMITZ, Ulrich (2016) „Kulturwissenschaftliche Orientierung in der Medienlinguistik.“ In: L. Häger/W. Holly/P. Krapp/S. Weber/S. Heekeren (Hrsg.), Sprache Kultur - Kommunikation. Ein Handbuch zur Linguistik als Kommunikationswissenschaft. Berlin/Boston: De Gruyter Mouton, 901-908.

URBÁN, Péter (2017) „Mehrsprachige Zeitschriften im Bratislava der Zwischenkriegszeit." In: C. Földes (Hrsg.), Interkulturelle Linguistik als Forschungsorientierung in der mitteleuropäischen Germanistik. Tübingen: Narr Francke Attempto, 241-250.

WÖLLSTEIN, Angelika/DUDENREDAKTION (Hrsg.) (2016) Die Grammatik: Unentbehrlich für richtiges Deutsch. Berlin: Dudenverlag.

ZHAO, Jin (2018) Wissenschaftsdiskurse kontrastiv. Kulturalität als Textualitätsmerkmal im deutsch-chinesischen Vergleich. Berlin/München: De Gruyter Mouton.

\section{Zusammenfassung \\ DIE DEUTSCHE ALLGEMEINE ZEITUNG: ANMERKUNGEN ZUR SPRACHE DER KASACHSTANDEUTSCHEN PRESSE}

Die Studie arbeitete zentrale sprachliche Profilmerkmale der Deutschen Allgemeinen Zeitung (DAZ) in Kasachstan heraus und lieferte dabei auch einen Beitrag zur Erschließung von Manifestationen der Kulturalität im analysierten interkulturellen Mediendiskurs. Der verwendete Ansatz war - im Denkrahmen der interkulturellen Linguistik - deskriptiv und vorrangig variations- bzw. kontaktorientiert. Die Besonderheit der Zeitung besteht vor allem darin, dass ihre Sprache praktisch monolingual deutsch ist, während ihr kulturelles Umfeld eine kasachisch-russische Prägung aufweist. Die daraus resultierende konkrete Sprachverwendung in der DAZ wurde unter Nutzung des Salienz-Konzeptes betrachtet. Im ausgewerteten Korpus des Jahrgangs 2017 wurden sprachbezogene Salienzen auf nahezu allen Ebenen herausgearbeitet, allen voran in Lexik und Phraseologie, Grammatik, Stil, Grafie sowie Typografie. Die eruierten Verwendungsbesonderheiten resultieren aus drei Prozessen typologisch unterschiedlicher Art: (1) aus prototypischen Sprachkontaktmechanismen, also aus Transfers oder Nachahmungen von Elementen, Strukturen und Modellen der Kontaktsprachen Russisch und Kasachisch; (2) aus Verfremdungsprozessen, z. B. Kontrastverschiebungen oder -übertreibungen, die sich aus einer unsicheren Beherrschung der Zielsprache Deutsch, etwa durch Übergeneralisierung des Sprachsystems, ergeben; (3) aus Normverletzungen aufgrund von Unachtsamkeit, d. h. Flüchtigkeitsfehler bzw. problematische oder grenzwertige Formulierungen, die selbst bei Textproduzenten mit exzellenter (nativer) Sprachbeherrschung vorkommen. Es ist nachgewiesen worden, dass quantitativ die mehrsprachigkeits-, kontakt- und/oder kompetenzbedingten Auffälligkeiten 
überwiegen, wobei sich der sprachliche Kontakteinfluss des Russischen als größer erwies als der des Kasachischen. Auf dieser Basis konnte der Beitrag die DAZ als eine mehrfach kulturasymmetrische Grenzgänger-Zeitung mit einer spezifischen Form von Mehrschriftlichkeit erfassen und beschreiben.

Schlüsselwörter: Mediendiskurs, Pressesprache, Mehrsprachigkeit, Sprachkontakt, Interkulturalität

\section{Abstract \\ THE DEUTSCHE ALLGEMEINE ZEITUNG: COMMENTS ON THE LANGUAGE OF THE KAZAKHSTAN-GERMAN PRESS}

The study presents some principal linguistic features of the Deutsche Allgemeine Zeitung (DAZ) in Kazakhstan and analyses culturally relevant elements in intercultural media discourse. Within the framework of intercultural linguistics, the approach used has been descriptive, primarily variation and contact-oriented. The newspaper analyzed is unique in that it is practically monolingual German, while culturally it is embedded in a predominantly Kazakh and Russian context. Language use in DAZ was examined on the basis of the salience concept. In the evaluated corpus of the 2017 volume, language-related saliences were found at almost all levels, above all in lexis and phraseology, grammar, style, and typography. Those findings result from three typological different processes: (1) prototypical mechanisms of language contact, therefore transfers or imitations of elements, structures and models of the involved languages in contact, Russian and Kazakh; (2) alienation processes, e.g. contrast shifts or exaggerations resulting from insecure proficiency of the target language German, for instance due to overgeneralization of the language system; (3) norm infringements as a result of inadvertency, meaning careless mistakes and problematic or borderline wordings, which occur even in text producers with excellent (native) language proficiency. It has been demonstrated that saliences due to multilingualism, contact and/or competence prevail, with the linguistic interference from Russian shown to be greater than that of Kazakh. On the basis of these findings, DAZ can be described as a cross-border newspaper, which is in many ways culturally asymmetrical, reflecting the multiliteracy of its text producers.

Keywords: media discourse, newspaper language, multilingualism, language contact, interculturality 
Povzetek

\section{DEUTSCHE ALLGEMEINE ZEITUNG - PRIPOMBE K JEZIKU KAZAHSTANSKONEMŠKEGA TISKA}

Študija obravnava osrednje jezikovne značilnosti časopisa Deutsche Allgemeine Zeitung (DAZ), ki izhaja v Kazahstanu, in raziskuje manifestacije kulturnosti v medkulturnem medijskem diskurzu. Ob predpostavkah medkulturne lingvistike je bil v študiji uporabljen deskriptivni in prvenstveno variantno- oz. stičnostnolingvistični pristop. Posebnost časopisa je predvsem $v$ tem, da je enojezičen, pisan le nemško, medtem ko je njegovo kulturno okolje kazahstansko-rusko. Ob upoštevanju teh dejstev je bila raba jezika v DAZ-u opazovana na osnovi koncepta salience. Izbrani korpus je zajemal letnik 2017, obravnavane pa so bile posebnosti na skoraj vseh ravninah, predvsem pa besedišče ter frazeologija, slovnica, slog, grafija ter tipografija. Ugotovljene posebnosti jezikovne rabe so posledica treh tipološko različnih procesov, in sicer: (1) prototipičnih jezikovnokontaktnih mehanizmov, torej transferjev ali posnemanja sestavin, struktur in modelov stičnih jezikov, ruščine in kazahstanščine; (2) potujitvenih procesov, npr. premikov ali potenciranja kontrastov, ki nastajajo zaradi negotovosti v obvladovanju nemščine kot ciljnega jezika in se kažejo kot posploševanje jezikovnega sistema; (3) kršenja norme zaradi nepazljivosti, t. j. napak zaradi površnosti oz. problematičnih formulacij, ki so na meji sprejemljivega in ki nastajajo celo pri avtorjih besedil z odličnimi jezikovnimi kompetencami (v maternem jeziku). Dokazano je bilo, da kvantitativno prevladujejo večjezičnostno, stičnostno in/ali kompetenčno pogojene posebnosti, pri čemer se je jezikovni stik z ruščino izkazal za vplivnejšega kot stik s kazahstanščino. $\mathrm{Na}$ tej osnovi je DAZ v prispevku označen in opisan kot večplasten kulturno asimetričen čezmejni časopis s specifično obliko večpisavnosti.

Ključne besede: medijski diskurz, časopisni jezik, večjezičnost, jezikovni stik, medkulturnost 